\title{
Naming and classifying old and new ECG phenomena
}

\author{
Adrian Baranchuk MD, Antoni Bayés-Genis MD PhD
}

See also pages 528, 529, www.cmaj.ca/lookup/doi/10.1503/cmaj.150816 and www.cmaj.ca/lookup/doi/10.1503/cmaj.150550

$\mathrm{T}$ wo linked reports present cases of atypical electrocardiography (ECG) patterns that represent manifestations of severe narrowing or occlusion of the proximal left anterior descending coronary artery. ${ }^{1,2}$ These ECG patterns, named after Wellens and de Winter, respectively, occasionally replace the classic ECG manifestations of ST-elevation myocardial infarction (STEMI). Initially described in $1982^{3}$ and $2008,{ }^{4}$ respectively, both correspond to proximal severe disease of the left anterior descending artery, with rapid recognition and intervention being required to ensure revascularization.

Certain commonalities in the clinical presentation, ECG evolution and required treatment lead us to believe that these ECG patterns may correspond to different manifestations of a single spectrum (Box 1). ${ }^{3,4}$ The differences between the two patterns may reflect the timing of ECG relative to total occlusion of the vessel, spontaneous reperfusion of the vessel, initiation of the ischemic process or resolution of the ischemic process. Recognizing the two patterns can help physicians to make correct and timely medical decisions, so as to rescue as much myocardium as possible. However, some inconsistencies have been noted in the literature, and these are reflected in the linked cases. ${ }^{1,2}$

These cases raise several questions: Are these true syndromes or simply ECG signs? Are they STEMI equivalents? Must all named ECG signs be present to use the term "Wellens syndrome" (or pattern) or "de Winter syndrome" (or pattern)? What distinguishes an ECG sign from a syndrome? These are not just semantic questions; rather, they are related to both medical knowledge and knowledge translation.

A syndrome should have both signs (e.g., the characteristic ECG pattern) and symptoms (e.g., chest pain). If the patient is pain-free at the time of evaluation, the physician should ask about prior history of angina to recognize one of these syndromes. Definitions that use terms such as "slight elevation" or "slight depression" are difficult to universalize; conversely, definitions that include "0.5-mV elevation or depression" can be difficult to measure and quantify. Finally, if a series of ECG signs must be present to define an ECG phenome- non, is the presence of only one or two of the signs enough to confirm the phenomenon? For example, in Wellens syndrome, lead aVR should show STsegment elevation. However, in the case presented by Das and Almajed, ${ }^{1}$ lead aVR was not elevated. Similarly, in de Winter syndrome, the total occlusion should occur in the proximal left anterior descending artery; however, in the case presented by Martínez-Losas and Fernández-Jiménez, ${ }^{2}$ the occlusion occurred after the first septal perforator, which indicates occlusion of the mid-third of the vessel. How strict should the definitions be for defining a new ECG phenomenon, and how strict should clinicians be in determining that particular cases belong to weakly defined syndromes?

In thinking about the inclusion of an ECG phenomenon in the literature, an example that comes to mind is Brugada syndrome, ${ }^{5}$ a rare genetic disease associated with sudden death in patients with apparently normal heart structure. The surface ECG remains the key to the diagnosis. Three different patterns were described initially, but the third pattern was removed from the definition in 2012. Despite their completely different morphologies, the two remaining types are simply called types 1 and 2 . The distinction between them is universally accepted, and thousands of papers can be found that address this rare genetic disorder.

The example of Brugada syndrome suggests that two distinctive patterns corresponding to the same physiopathologic origin (i.e., occlusion or severe narrowing of the proximal left anterior descending artery) should not be named differently, given that the authors describing the patterns belong to the same academic group. It would be easier to learn and to teach these patterns if, for example, the phenomena were to be called Wellens ECG patterns 1 and 2. In fact, the de Winter ECG

\section{KEY POINTS}

- The electrocardiographic patterns of Wellens syndrome and de Winter syndrome should be recognized promptly as possible presentations of acute coronary syndromes, to allow for timely investigation and revascularization of the proximal left anterior descending coronary artery.

- A systematic approach for development of new electrocardiographic terminology and eponyms is suggested to optimize learning and teaching strategies and to facilitate consistency in the literature. 


\begin{tabular}{|lll|}
\hline Box 1: Characteristics of Wellens syndrome and de Winter syndrome 3,4 & \\
\hline Characteristic & \multicolumn{1}{|c|}{ Wellens syndrome } & de Winter syndrome \\
\hline Prevalence as a STEMI equivalent, \% & 18 & 2 \\
\hline Chest pain & Intermittent & Present \\
\hline Troponin & Slightly elevated or normal & Elevated \\
\hline ST segment in precordial leads & Slightly elevated & Depressed \\
\hline Preferential precordial leads & V1-V6 & V2-V4 \\
\hline T-wave characteristics & Profoundly negative or biphasic and symmetric & Positive and symmetric (hyperacute T waves) \\
\hline T-wave morphology & Abrupt take-off of the negative T wave & Ascending limb of the T wave commencing \\
& from V2 to V4 & below the isoelectric baseline \\
\hline Precordial R-wave progression & Normal & Normal \\
\hline Pathologic Q waves & No & No \\
\hline ST-segment elevation in lead aVR & No & Yes \\
\hline Angiographic findings & Critical narrowing of proximal LAD & Occlusion of proximal LAD \\
\hline Evolution of the ECG pattern & May evolve into "classic" STEMI within weeks & Stable or evolving into "classic" STEMI \\
\hline Note: ECG = electrocardiography, LAD = left anterior descending artery, STEMI = ST-segment elevation myocardial infarction. \\
\hline
\end{tabular}

phenomenon was named after the first author of a report of a relatively small series of 30 patients. If this were the general rule for such observations, clinicians would have to remember the names of more than 100 new ECG phenomena reported in the past decade, which would be impractical.

For an ECG phenomenon to gain universal recognition deserving of an eponym in the literature, we suggest that the following conditions should apply.

First, there should be multiple independent reports (i.e., visibility). The phenomenon in question should be seen, recognized and reported by several groups, preferably internationally. There will be a period when the terminology is inconsistent, until multiple reports of the phenomenon have appeared and practitioners in the field can agree on an appropriate name. One example is early repolarization syndrome, which has also been termed early repolarization pattern, early repolarization variant and Haïssaguerre syndrome.

Second, a clear physiopathologic basis should be apparent. It is clear that the common pathway for both syndromes reported in the linked articles is severe narrowing or total occlusion of the proximal left anterior descending artery; however, there is little speculation in the literature about why the ECG patterns are so distinctive. Specifically, how can the electrical activity of the heart and, more specifically, its repolarization differ so much from one pattern to another? Is it a marker of the disorder's evolution or an indication of reperfusion? This requires further exploration.

Third, clinical reproducibility should be demonstrable, on the basis of careful investigation. For example, one would expect that, in cases in which the left anterior descending artery is occluded prox- imally and the ECG depicts the de Winter pattern, the pattern should reappear if the artery is opened and subsequently becomes re-occluded. Such a scenario is not rare in the context of acute coronary syndromes, so a review is needed of all Wellens and de Winter cases to check for reoccurrence of the pattern upon re-occlusion.

Fourth, experimental models should be established. Several animal models of ischemia exist, but a model suitable for reproduction of these phenomena is lacking. An experimental model would help advance knowledge and would allow better discussion of the temporal relation of the ECG phenomenon to the occlusion or to the initiation of myocardial ischemia.

In conclusion, we call for a systematic approach to the naming and inclusion of new ECG phenomena in the already extensive literature.

\section{References}

1. Das D, Almajed NS. Wellens syndrome. CMAJ 2016;188:529. Martínez-Losas P, Fernández-Jiménez R. de Winter syndrome. CMAJ 2016;188:528

3. de Zwaan C, Bär FW, Wellens HJ. Characteristic electrocardiographic pattern indicating critical stenosis high in left anterior descending coronary artery in patients admitted because of impending myocardial infarction. Am Heart J 1982;103:730-6.

4. de Winter RJ, Verouden N, Wellens H, et al. A new ECG sign of proximal LAD occlusion. N Engl J Med 2008;359:2071-3.

5. Bayés de Luna A, Brugada J, Baranchuk A, et al. Current electrocardiographic criteria for diagnosis of Brugada pattern: a consensus document. J Electrocardiol 2012;45:433-42.

Affiliations: Division of Cardiology (Baranchuk), Queen's University, Kingston, Ont.; Servicio de Cardiologia (Bayés-Genis), Hospital Germans Trias I Pujol, Badalona, Catalunya, Spain.

Contributors: Adrian Baranchuk conceived the article, wrote the first draft, suggested references and revised the final draft. Antoni Bayés-Genis conceived the article; supervised, modified and corrected the first draft; and wrote the second and final drafts. Both of the authors approved the final version for publication and agreed to act as guarantors of the work. 